\title{
Jahresbericht über die Philosophie im Islam. Von
}

\author{
Prof. Dr. Horten in Bonn.
}

(Fortsetzung.)

Horten, D. M., Die philosophischen und theologischen Ansichten von Lahígi ca. 1670 nach seinem Werke: „Die aufgehenden Sterne der Offenbarung" einem Kommentare zur Dogmatik des Tusi (tagríd) 1273 †.

Lahígi ist ein orthodoxer Theologe. Um so interessanter ist es, bei diesem den Einfluß der griechischen Philosophie und liberaler Lehren festzustellen. Es ist nun eine offenkundige Tatsache, daß er ganz und gar von gaiechischer Philosophie beherrscht ist und auch offen für dieselbe eintritt, obwohl er sich dadurch in eine oppositionelle Stellung zur altorthodoxen Theologie, d. h. zu den Traditionen seiner eigenen Schule setzt. Das Eindringen und Obsiegen des griechischen Geistes in die islamische Theologie ist also noch bei diesem späteren Vertreter derselben zu beobachten. Seit Avicenna ist dieses das allgemeine Entwicklungsgesetz islamischen Geisteslebens, das man in seinen Phasen in den verschiedenen Jahrhunderten feststellen kann. Die Nachrichten, die Lahígi selbst in seinem Werke gibt, erlauben es, sogar bei den großen Gegnern Avicennas, Rázi und Taftazáni dieses selbe Gesetz wirken zu sehen. Sie bekämpfen Avicenna nur in einzelnen seiner Thesen, bewegen sich dabei aber durchaus im Banne griechischer Gedanken. Noch viel deutlicher tritt dieses Entwicklungsgesetz bei den Ävicenna freundlich gesinnten Theologen auf. Die intelessante Tatsache ist vor allem zu konstatieren, daß der "Heide“ Avicenna von orthodoxen Theologen des Islam gegen die Angriffe anderer Theologen verteidigt wird. Eine der auffälligsten Beispiele für jenes Gesetz ist schließlich Lahígi, der eine strenge Orthodoxie vertritt, dabei sich aber so sehr den Gedanken Avicennas nähert, daß man einen unmittelbaren Schüler dieses Meisters zu hören glaubt. Seine Lehren übel die Wissenschaft, das Sein und dessen Modi, die Ursachen, das Wesen der Naturdinge (Hyle und Form), die Gesetzmäßigkeit im Naturgeschehen, das Wirken Gottes, den Nus usw. sind durchaus in griechischem Geiste gehalten ${ }^{1}$ ).

1) Das Werk Schahristanis: „Die Bekämpfung der griechischen Philosophen" (musàraat alf.) findet sich hier S. 31 und auch bei Schirázi unter dem Titel: Der "Totschläger der griech. Philos." (musári’alf. bei i. Hall. almusàraàt). - vgl. ferner: Kantstudien XVII 483. 
Horten, Die spekulative und positive Theologie des Islam nach Kázi $1209 \uparrow$ und ihrc Kritik durch Tusi 1273 nach Originalquellen übersetzt und erläutert mit einem Anhang: Verzeichnis philosophischer Termini im Arabischen, Leipzig 1912.

Mit dieser Veröffentlichung werden der vergleichenden Religionswissenschaft Dokumente vorgelegt, die im Mittelalter des Islam für lange Zeit und breite Kreisc die Theologie ausmachten. Sie wurden wegen ihrer klaren und kurzen Form als Kompendium der philosophisch orientierten Theologie bcnutzt. Aus ihnen ersieht man, welche Probleme für die höher gebildeten Muslime im Zentrun des Interesses standen und wie sie behandelt wurden. Die Stellung und Behandlung der Probleme ist eine durchaus philosophische. Nach Avicenna und Gazali sind alle maßgebenden Theologen im Islam in der griechischen Philosophie ausgebildet oder wenigstens über sie genügend orientiert. Sie kennen ihren Altmeister Avicenna von Grund auf und wenn sie auch in einzelnen Fragen gegen ihn Stellung nehmen - dieses berreist ihre geistige Selbständigkeit - bewegen sie sich doch durchaus in den Bahnen des philosophischen Denkens. Mit Rázi ist der Punkt der Entwicklung gegeben, in dem die große Masse der gebildeten Muslime sich mit der griechischen Philosophie zu befassen anfängt, zuerst aber noch mit einigem Zagen und manchen Polemiken gegen das "Heidnische“ in Avicenna zu Werke geht, um in der weiteren Entwicklung (z. B. Igi und Gurgani) sich dem philosophischen Denken unbefangener zu öffnen.

Die Kritik Tusis zeigt deutlich, daß das Verständnis der griechischen Philosophie in theologischen Kreisen im XIII. Jahrhundert im Fortschreiten begriffen ist. Rázi ist vielfach ganz unsicher. So versagt er in der Erklärung des Wissens Gottes (S. 57), schwankt selbst in seinen Begriffen (z. B. der Definition des Wissens S. 57 Z. 4 unt. u. Anm.), unsicher in seiner Beweisführung und mehr auf das Logisch-Formale sehend (S. 58, 6 unt. u. passim), während Tusi die Gedanken sachlich klarer und tiefer faßt (S. 63, 9f., wo Rázi einen Fehlschluß in aliud genus, ex ordine logico in ordinem realem macht, 59, 5 unt.: Das Prinzipielle betonend; 58, 10 unt. usw.). Allerdings begeht auch Tusi ein Versehen, indem er die Möglichkeit eines Instrumentes der Schöpfung damit widerlegt, daß er die Frage nahezu zu einer Wortfrage macht (S. 35 A. 1), was sie in der Tat nicht ist. Die Korrekturen, die Tusi (passim) an den Äußerungen Rázis über die griechischen Philosophen vornimmt, sind gerade für das Besserwerden des Verständnisses der griechischen Gedankenwelt beachtenswert. Das auch für das Verständnis der älteren Theologie ein Gewinn erzielt wird, zeigt die Behandlung der Modustheorie des abu Háschim, die deutlich ein Inhaerens und das Inhaerenzverhältnis unterscheidet ( $S^{\prime}$ 63, 2f. u. ib. Z. 28, ferner 72 Mitte), ersteres sogar als etwas der Gottheit nicht „Anhaftendes" bezeichnet. Jene uns fremde Gedankenwelt (im Grunde die Lehre der Vaischesika von der Inhaerenz) wird dadurch in wünschenswerter Weise beleuchtet. An der Oberfläche haften ferner die Bewejse Rázis, daß das formelle Objekt des Sehens das Sein ist (70). Vielleicht könne das des Hörens einen gleichweiten Umfang haben. Die Hörbarkeit des innergöttlichen Wortes und visio beatifica soll damit als annehmbar erwiesen werden. Die 
Kritik Tusis nimmt sich dagegen sehr kühl und nüchtern aus. Ähnliche Ob̉erflächlichkeiten begeht Rázi 75-76 (duo agentia in unum obiectum), 76 Mitte, 77 unt. (die Konsequenzen der Handlung), 81 (naive Antwort auf die bekannte Schwierigkeit gegen die freie Handlung betr. den sensus compositus und divisur) usw. Aus deni vorliegenden Werke läßt sich also derjenige Geist charakterisieren, der den Kampf-gegen mehrere Thesen Avicennas unternommen hat. Die Verteidiger der ǵriechischen Philosophie haben-sich .von nun an gegen Rázi zu wenden und seine Aufstellungen zu erschüttern. Wie leicht ihnen dieses gelingt und wie sicher und überlegen sie sich ihrer Sache fühlen, zeigt - Tusi. Noch schärfere Kıitik üben spätere Theologen z. B. Kuschgi, Schirazi . $(1640 \dagger)$, Lahígi usw. Im ganzen kann man sagen: Es ist der islamischen spekulativen Theologie und Philosophie nicht schwer gefallen, die Bedenken Rázis abzuwehren und dem griechischen Geistesleben die Bahnen, auf denen es in den Islam eindrang, offen zu halten.

Die Übersetzung des Kompendium (muhassal) Rázis (vgl. die Rezension: Archiv XX. Bd. 1907 S. 411f.), dieses Merksteins der islamischen Philosophie, ist nunmehr beendet. Die allgemein philosophischen Teile sind in dem Werke wiedergegeben: Horten: Die philosophischen Ansichten von Rázi und Tusi (Bonn 1910), die Aufsührungen über die Modustheorie in ZDMG. Bd. 63 S. 303 bis 324. Danach waren noch die theologischen Teile zu geben (von S. 106 des arab. Teiles), die hier also vorliegen. Sie bilden ein größeres Ganze mit meinen iibrigen Veröffentlichungen über islamische Theologie (,Die philosophischen Probleme“, „Rázi und Tusi“, „Abu Raschíd“ und „Die philosophischen Systeme"), die Bausteine zu einer allseitigen Darstellung der philosophischen Ideenbildungen der ersten Zeit des Islam sein wollen. Die philosophische Beurteilung der einzelnen Lehren und die Wertung der Gedanken Rázis im Vergleiche zu Tusi und anderen vom logischen und ideologischen Standpunkte ist eine Aufgabe, die erst nach eingehenden Vergleichen mit früheren und späteren Systemen in historischem Sinne erledigt werden kann.

Der Anhang bringt eine Zusammenstellung philosophischer Termini in Arabischen. Er zerfällt in zwei.Teile, das eigentliche Lexicon und die Terminologie der „Thesen" Avicennas, die mit den „Definitionen“ Gurganis und den Ausdrücken der „Erlösưng der Seele“ (eines Werkes Avicennas) als besondere Einheit zusammengefaßt wurden. Die Berechtigung zu dieser Gruppierung hat Forget in seiner Ausgabe der "Thesen" bereits ausgesprochen. Avicenna. ist in denselben bestrebt, den Anfänger in die Philosophie einzuführen und besonders die Terminologie zu fixieren. In der historischen Entwicklung sind sie in der Tat auch zu einem Maßstabe der philosophischen Ausdrucksweisen geworden. Eine verwandte Tendenz verfolgen Gurgani und Arabi in ihren Lexicis. Es ist daher berechtigt, diese als die Grundlagen der philosophischen Terminologie gesondert für sich zu betrachten.

Es ist eine anerkannte Tatsache, daß die gesamte höhere Geisteskultur des Islam ron philosophischen Voraussetzungen durchdrungen und getragen wird. Wollen wir uns daher dieses eigenartige Geistesleben in der Weise vergegenwärtigen, in der es einst wirklich war, und wollen wir es so nachdenken, wie es gedacht wurde, dann können wir dic ihm zugrunde liegenden philo- 
sophischen Gerlanken nicht außeracht lassen. Der Kinfluß der Philosophie crstreckt sich im Islam nicht nur auf die Theologie, die Koranexegese und Traditionswissenschaft, sondern auch auf die ('eschichte (ibn Haldán), die Naturwissenschaften (besonders in Einleitungen und allgemeinen Erörterungen), die Medizin (\%. B. die „Allgemeinhegriffe der „Medizin“, ein Werk Avicennas), die Mystik, Ethik und sogar die Poesie, insofern sie das eine oder andere dieser (iebiete ( $\%$. B. die Mystik) berührt. Daher ist es im Interesse der Erschließung der islamischen Geisteskultur berechtigt, die philosophischen T'ermini, deren Klarstellung mit besonderen Schwierigkeiten verbunden ist, eingehend $\mathrm{zu}$ behandeln.

Nicholson, Reynold A., The Kashf. al-Mahjúb by 'Alí b. 'Uthmán al Jullábí al Hujwírí in: E. J. W. Gibb Memorial Series. vol. XVII, 1911, S. XXIV + 443 , gr. $8^{0}$.

In dem vorliegenden stattlichen Bande erschließt Nicholson, der Meister in der Kenntnis der islamischen Mystik, weiteren Kreisen den ältesten mystischen Text der persischen Literatur. Der übersetzte Text: „Die Enthüllung des Verschleierten" ist ein späteres Werk des Gaznawi (ca. $1073 \dagger$ ). Er gibt eine historische und dogmatische Darstellung der Mystik im Islam. Diese eigenartige Form der Erlebnis- und Gefühlsreligion ist sowohl in ihrem Asketismus als auch ihrer pantheistischen Spekulation (die die Welt als Phänomen auffaßt und Gott als das eigentliche Sein, der Realgrund der Phänomene) ein indischer Einfluß, der sich in koranische Formeln zu kleiden sucht. Im Islam selbst hat er jedoch eigenartige Umgestaltungen erfahren, wie die Lehren der einzelnen Meister, die Gaznawi aufzählt, zeigen. Jeder schildert in seiner Weise das ihm eigene religiöse Erlebnis und gelangt dabei vielfach $\mathrm{zu}$ besonderen dogmatischen und ethischen Aufstellungen. Trotz der harmonisierenden und apologetischen Tendenz des Gaznawi leuchtet deutlich durch, da $B$ in der mystischen Strömung zwei Richtungen geltend waren, eine extreme - die ausgesprochen pantheistische - und eine gemäßigte, die die pantheistischen Ideen als heterodox ablehnte. Letzterer gehört Gaznawi an. Nach ihm ist die Substanz des menschlichen Ich unvergänglich. Sie wird also auch im Nirwana nicht von Gott absorbiert. Die menschlichen Eigenschaften fallen jedoch bei dem Versinken in die Gottheit der Vernichtung anheim. Durch die Lehre von diesem gemäßigten Nirwana sucht Gaznawi buddhistisches und islamisches Gedankengut zu harmonisieren. - Der Mensch besteht nach Gaznawi aus vier Teilen: dem Geiste (sirr), dem Pneuma, der sensitiven Seele und dem Leibe (S. 309; S. 199 werden nur drei aufgezählt). Diese bilden konzentrische „Sphären“, deren innerster Kern der Geist und deren äußerste Schale der Leib ist. Die Ewigkeit des Lebensgeistes, eine weitverbreitete Lehre (S. 266) wird von Gaznawi entschieden abgewiesen. $\mathrm{Er}$ ist sehr bestrebt seine Orthodoxie hervorzuheben, besonders auch durch seine Polemik gegen die liberalen Theologen.

Die Zusammenstellung mystischer Termini S. 367ff. ist als Ergänzung der bekannten mystischen Lexika von Arabi 1240 †, Káschi (Abdarrazzák) $1330 \dagger$ und Gurgáni $1413 \dagger$ sehr dankenswert. Sie zeigt, wie mystische Aus- 
drücke durch Schultradition zu sehr abliegenden Bedeutungen gelangen können. Von der philologischen Kritik darf also dagegen kein Bedenken erhoben werden, daß man diese Termini „freier" übersetzt, ohne sich an die Grundbedeutung zu enge anzuschließen; denn diese „freiere“ Übersetzung gibt den Gedanken wieder, den der Autor ausdrücken will, während ein Festhalten an der Grundbedeutung einen anderen Gedanken wiedergeben würde; also eine geradezu fehlerhafte Übersetzung bedeutete. Tauhid = unification (385,5 unt.) bezeichnet z. B. vielfach schlechthin Gott (Bekenntnis und Preis der Einheit Gottes) und sogar den Gedanken, daß Gott die Universalursache des Weltalls ist. Der eigenartigen Biegsamkeit der arabisch-pelsischen Termini ist Nicholson in der Weise trefflich gerecht geworden, daß er in kritischen Fällen eine entsprechend freie Wiedergabe findet ${ }^{1}$ ). Die Haschwíja, über deren Lehren ich in dem Buche: Die philosophischen Probleme der spekulativen Theologie 1910 mehrere Berichte beibringen konnte (vgl. auch Zeitschr. f. Assyr. 1911 Bd. XXVI S. 196) treten bei Gaznawi als Anthropomorphisten

$\left.{ }^{1}\right)$ Statt Sulami (Azdi $1021 \dagger$ ) ist nunmehr mit Mart. Hartmann Or. L. Ztg. 1912 Sp. 128 Sullami, der Begründer der Lehre von der mystischen Stufenleiter, Klimax zu lesen: Die Termini sind durchgängig diejenigen der Philosophen: hudút (,origination“ S. 280): zeitliches Entstehen, muhdat (phänomenal; 270) : zeitlich entstanden, tasdík (verification; 286): Fürwahrhalten, "Glauben", sirr (heart; 309 u. oft): reiner Geist, der abstrakt denkt und Gott erschauen kann, kaifijat (nature; 308): Eigenschaft in Gegensatz zur Substanz, aijárán (hidden spiritualist; 100): Mystiker die jegliche Kultur veispotten (und vielleicht ganz unbekleidet umhergehen vgl. 'ar - nach indischem Vorbilde), gama 'a bain aššari 'ah wal hakikah (he combined the Law and the Truth; 139): er betätigte sich gleichzeitig in der praktisch-ethischen wie auch der theoretisch-kontemplativen Seite der Religion, 'ain (the true idea; 149 unt.): die konkrete Wirklichkeit („,das Individuum“), baká (subsistance; 185), vielfach: „unvergängliche Dauer", S. 204: the qualities of a horse are altered by ,mortification“, doch wohl „Dressur" (wenn auch der arabische Terminus für beide derselbe ist), mugassam (incarnate; 224) : mit einem Körper ausgestattet (auch ohne Inkarnation), intikál (transmigration; 236): räumliche Bewegung (die für ein unkörperliches Wesen wie Gott undenkbar ist (und tagzia = Zusammensetzung aus Teilen), huwija (the Absolute; 238): d i e Individualität (die absolut besteht, also Gott - mit pantheistischen Grundgedanken!), máhall i hawadit (locus of phenomena; 244): Substrat zeitlich entstehender Akzidenzien (dlas der Veränderung unterworfen ist), ta'til (denial of the Creator; 257, 1) vielleicht besser: Thesis, daß Gott keine Einwirkung auf die Welt ausübt (Wurzelbedeutung: müßig, tatenlos sein), die Bahshami sekt (295) sind die Anhänger des abu Háschim $933 \dagger$, der mehrmals genannte Sulami ist unter dem Namen Azdi $1021 \dagger$ bekannt. Die Verzeichnisse f. $421 \mathrm{ff}$. sind sorgfältig gearbeitet (Bangári fehlt). Eine chronolog ische Zusammenstellung der genannten Mystiker würde den geschichtlichen Überblick erleichtert haben. 
auf, was mit ihrer anderwcitig bekannten intellektuellen Rückständigkeit übercinstimmt.

Folgende sind die Themata der Mystik, die Gaznawi von dem Standpunkte einer durchaus idealistischen und durchgeistigten Lebensauffassung aus behandelt: Gotteserkenntnis, Wert der Armut, mystische Reinheit, das Mönchsgewand (Bettlergewand, ein Symbol der Weltentsagung), das Streben, von den Menschen verachtet und getadelt zu werden, Glaube, Reue, Geselligkeitstugenden, kurz: Regeln für jede Betätigung des Lebens. Möge dieses Werk zum Verständnisse islamischer Gedankenwelt beitragen.

Nicholson, A. R., The Tarjumán al-Ashwáq, a collection of mystical odes by Muhyi'ddín ibn al-Arabí; London Royal Asiatic Society 1911; VII + 155 S. $8^{0}$.

Arabi $1240 \dagger$ gilt als der bedeutendste Mystiker im Islam. Seine Gedankenführung und Weltanschauung kennen zu lernen ist daher ein lebhafter Wunsch mancher Forscher. In seinen hier vorliegenden Gedichten scheinen seinen philosophischen Lehren nur stellenweise durch, und ohne den beigegebenen Kommentar, den Arabi selbst zu seinen mystischen Liebesgedichten verfaßte, wären deren tiefere Gedanken wohl unerkennbar geblieben. Arabi ist ein pantheistischer Mystiker. Die Welt ist die Summe der Differenzierungen der göttlichen Substanz, d. h. „der Namen Gottes“. Das Glück des Menschen besteht in dem Aufgehen in der Gottheit, dem Nirwana. Das Weltall stellt sich in drei Stufen dar, dem Weltintellekte, der niederen, sublunarischen und der zwischen beiden liegenden mittleren. Welt. Diese vermittelt die Einwirkungen von der Geisteswelt zur niederen Welt und ist mit der Natur des sensitiven Prinzipes ausgestattet zu denken. Der Lebenskampf besteht in einem Streben nach dem Geistigen, den Visionen des Göttlichen in der jenseitigen, unmateriellen Welt. Alle Religionen sind gleichwertig. In der Religion der Liebe werden alle zu einer höheren Einheit und zu wahrhaft edler Toleranz zusammengefaßt. Jede Religion ist eine berechtigte Offenbarungsform der einen großen Weltreligion. - Indische und neuplatonische Lehren durch: dringen sich hier zu einer schönen Harmonie.

Mit diesem Mystizismus berührt sich in manchen Punkten die Philosophie der Babí-Beháí-Sekte. Sie gibt dem Neuplatonismus mehr Spielraum und macht gegen den extremen Pantheismus Front. Die Literatur über diese moderne Erweckungsbewegung im Islam und ihre Geschichte findet man übersichtlich zusammengestellt und mit Verständnis erläutert von Römer, Dr. Hermann: Die Bábí-Beháí. Potsdam 1912. Drei dieser Gedichte des Arabi wurden in deutscher Übertragung mit Kommentar'und orientierender Einleitung besonders herausgegeben: Horten: Mystische Texte aus dem Islam. Drei Gedichte des Arabi. Bonn 1912. S. 18. Die Einleitung bespricht die drei Hauptzweige der islamischen Mystik (den christlichen, griechischpersischen und indischen), die Weltanschauung Arabis und das Wesen der mystischen Intuition, der mit der Seinslehre der Philosophen (Kontingenzbeweis) verglichen wird. Vgl. Theol. Litrtztg. 1912 Sp. 450. 
Ikbal, Shaikh Muhammad, The Development of Metaphysics in Persia: a contribution to the history of muslim Philosophy; London 1908. $\mathrm{XII}+195 \mathrm{~S}$.

Der Verfasser bemerkt in der Einleitung, die Perser seien nur für eine oberflächliche Betrachtung der Dinge geeignet. Glücklicherweise trifft diese Behauptung in ihrer naiven Allgemeinheit nicht zu, leider bewahrheitet sie sich aber bei dem Verfasser selbst. Sein Buch ist von der Psychologie eines 18-20jährigen Knaben aus geschrieben: In den Kreis der persischen Philosophie sind hineingezogen die Lehre des Zaroaster, der Manichaeismus, die islamisch-theologischen Streitigkeiten, das Ismaëlitentum usw. also alles was Weltanschau ung bedeutet und zwar mit großem Rechte. Dabei laufen allerdings die allerschwersten Mißverständnisse unter, die aufzuzählen überflüssig ist, da das Buch keinen Anspruch darauf erheben kann, wissenschaftlich ernst genommen zu werden. Da der Verfasser manche noch unbekannte Quellen heranzieht, bringt er einiges Neue, das aber jedesmal nachzuprüfen sein wird. Psychologisch ist dieser Essay in gewissem Sinne interessant. Er zeigt, wie die Psychologie eines Orientalen, die scheinbar eine „,moderne Bildung“ erlangt hat, in wissenschaftlichen Fragen reagiert.

Die Mystik nimmt in der Darstellung einen sehr großen Raum ein, ein Beweis für die Wichtigkeit, die ihm von persischer Seite beigelegt wird. Die europäischen Darstellungen von der Gesamtkultur des Islam müssen diesen Tatsachen Rechnung tragen. In der Erklärung dieses die orientalische Kultur so tief ergreifenden Phaenomens der Mystik unterscheidet. Ikbal das tiefer liegende Wesen von den äußeren Erscheinungs- und Darstellungsformen. Die europäische Wissenschaft habe nur auf das Äußere geachtet, wenn sie vermeint, mit dem Hinweisen auf Neuplatonismus, indische oder altarabische Gedanken das innere Wesen der persischen Mystik. begreifen zu können. Dieses ist vielmehr in den psychologischen, politischen und sozialen Verhältnissen der Zeit zu suchen, die zur Weltflucht, Askese und Versenkung in Gott (dem Weśen der Mystik) hintreiben mußten. Dieselben Gesichtspunkte werden auch von Prof. Mart. Hartmann (Berlin) zur Erklärung der islamischen Kultur mit Glück und guten Gründen betont. Ob dieser im Zeitcharakter liegende Hang zur Weltflucht und Flucht ins Jenseits nun nouplatonische oder indische Gewandung anlegt, ist für sein Wesen durchaus gleichgültig. Der Nachweis dieser äußeren Einflüsse erklärt also fast nichts und wendet die Aufmerksamkeit auf Nebensächliches. Sicher ist an dieser beachtenswerten Auffassungsweise, daß ein so tiefliegendes und delikates Problem wie das religiöse Leben der mystisch Denkenden und Empfindenden nicht als hineingetragene äußere Kultureinflüsse restlos verstanden werden kann. Sehr im Recht ist Jkbal auch, wenn er der Ansicht entgegentritt, daß mit Avicenna und Gazali die islamische Philosophie zu Ende gegangen sei. Es ist leicht verständlich, daß $\mathrm{ihm}$, dem Orientalen, der mit der orientalischen Gedankenwelt in engerem Kontakt steht, wie ein Europäer, die genannte Ansicht als etwas Ungeheuerliches erscheint. Fällt doch die philosophische Bewegung in ihren breiten Strömen erst in die Zeit nach Gazali. - Würde der Verfasser sich möglichste Beschränkung in allgemeinen Beurteilungen und 
Thorblicken auferlegen sad soine Tätigkeit auf die Herausgabe cinzelner Quellen konzentricron, dann könnte er der Wissenschaft sicher noch manche Dienste leisten. (Fin anderes Urteil s. unten sub No. 125.)

Von islamischer Philosophie ist in einer Studie von Dr. S. Horovitz: Die Psychologie des Aristotelikers ibn Daud (Breslau 1912, Jahresbericht des jüdisch-theologischen Seminar's für das Jahr 1911) viel die Rede. Die Denkweise dieser jüdischen Philosophen ist durchaus identisch mit der Avicennas. Die Ausführungen des Verfassers geben ein klares Bild der psychologischen iehren des i. D. Von einzelnen Ungenauigkeiten abgeschen bedeutet diese Arbeit einen dankenswerten Beitrag zur Geschichte der mittelalterlichen Philosophie, die eine für Christentum, Judentum und Islam in den Grundzügen gemeinsame Gedankenwelt darstellt, in der dem Islam zweifellos der Vorrang zukommt ${ }^{1}$ ).

Sauter, Constantin: Avicennas Bearbeitung der aristotelischen Metaphysik. Freiburg, Herder, 1912. S. 114.

Die islamische Philosophie stellt der geschichtlichen Forschung die Aufgabe, ihren Werdegang aus der griechischen und ihren Übergang zu der lateinischen-mittelalterlichen Philosophie aufzuklären. $\mathrm{Zu}$ dem letzten Teile dieser Aufgabe will S. einen Beitrag liefern, indem er zu seiner Schilderung Avicennas sich ausschließlich auf die den Scholastikern bekannten, la teinisch en Übersetzungen stützt unter Nichtbeachtung der modernen Übersetzung (Horten: Die Metaphysik Avicennas; Halle 1907) und anderer orientalistischer Arbeiten. Die bedenklichen Konsequenzen dieser Arbeitsmethode zeigen sich jedoch fast nur in den einleitenden Kapiteln (auf fast jeder Seite mußte ich Fehler notieren), während die philosophischen Ausführungen des zweiten Teiles, also das Wesentliche. sehr zutreffend sind. Dadurch ist der Beweis geliefert, daß auch Nichtorientalisten bis zu einem gewissen Grade an der Lösung der genannten großen Aufgabe mitzuarbeiten berechtigt sind. Die. wichtigsten philosophischen Lehren sind $j a$ auch in den lateinischen Übersetzungen erkennbar und können daher auf Grund dieser geschichtlich (Trennung des aristotelischen Kernes von den späteren Weiterbildungen) behandelt werden. Es muß jedoch betont werden, daß das Bild des lateinischen Avicenna in manchen Zügen von dem des arabischen verschieden ist. Es hat den Anschein, daß Avicenna in seiner lateinischen Gestalt kein gläubiger Muslim war und die Lehre von der doppelten Wahrheit gelehrt habe ${ }^{2}$ ). Nichts

1) Die Lehren des i. Daud erinnern sogar in ihrem Wortlaute stark an Farabi und Avicenna. Neues ist bei i. D. nicht zu verzeichnen. Viele polemische Bemerkungen des Verfassers hätten fortbleiben müssen. An diesem Orte ist ferner zu nennen: Horovitz, Dr. S., Die Stellung des Aristoteles bei den Juden des Mittelalters. Ein Vortrag. 18 S. 1911. In: Schriften, herausg. von der Gesellschaft zur Förderung der Wissenschaft des Judentums.

2) Dabei geht es nicht ohne Widersprüche ab. S. 111 erkennt S. auf Grund unzweideutiger Texte an, daß (Muhammad) der Prophet eine übernatürliche, alles menschliche Maß nicht nur graduell, sondern wesentlich überschreitende 
liegt dem arabischen Avicenna ferner als dieses. - Gott erscheint unserem Philosophen (S. 89, 15) nicht nur als der erste Beweger - bei Averroes tritt dieser Gedanke in den Vordergrund -, sondern als der Verleiher des Seins (almûgid). Auch Averroes hat in seiner lateinischen Gestalt das Schicksal erlebt, zum Freidenker gestempelt zu werden, obwohl gerade er mit besonderer Ängstlichkeit an der Orthodoxie des Islam hängt....Daß Gott die materiellen Individua nach ihrer ganzen individuellen Wirklichkeit erkennt, lehren beide sehr deutlich. In einem Punkte scheint mir das frühe Mittelalter eine bessere Kenntnis als das spätere zu haben. Wie bereits de Boer 1894 gefunden hat (Die Widersprüche der Philosophie S. 63, 101-103), ist Averroes Pantheist oder hat wenigstens solche Tendenzen. In der Zusammenstellung des Averroismus mit den pantheistischen Lehren eines Amalrich und David in der Verurteilung ron 1215 (S. 23-25) könnte eine richtige Tradition hierüber liegen.

Den wenigen Orientalisten kann man es nicht sehr zum Vorwurfe machen, daß sie die philosophische Literatur etwas außer acht gelassen haben. Wohl aber kann man es den sehr zahlreichen Gelehrten, die sich mit der Geschichte der mittelalterlichen Philosophie :befassen, verargen, daß sie die lateinischen Übersetzungen der islamischen Philosophen bisher noch nicht in textkritischer Weise ediert haben. Wenn dadurch auch für die Textkritik des arabischen Originals nichts besonderes Wertvolles erzielt werden sollte, so würde dadurch doch der Einfluß der Muslime auf das Abendland verständlicher werden. Zugleich müßten die Zitate aus den muslimischen Philosophen bei den Scholastikern gesammelt und untersucht und vor allem der Einfluß jener auf die Lehren der christlichen Philosophen klargestellt werden.

Als Ganzes ist die Arbeit Sauters sehr zu begrüßen. Sie bedeutet eine Einführung in die scholastisch-muslimischen Beziehungen im Mittelalter. Die leider sehr zahlreichen Fehler sind dem gegenüber zu entschuldigen. Sie betreffen meistens nur solche Punkte, die sich auf orientalistische Fragen und den originalen, arabischen Avicenna beziehen. Zu dessen Verständnis ist die Kenntnis der islamischen Umwelt erforderlich, die zur Zeit Avicennas eine ganz erstaunliche, an moderne Verhältnisse erinnernde Höhe erreicht hatte, was aus der Biographie Guzgánis und seiner Einleitung zur ,Genesung der Seele“ hervorgeht. (Vgl. meine Rezensionen desselben Werkes in ZDMG. 66, 175 ff. Theol. Litztg. 1913 No, 6 Sp. 173. Zeitschr. f. Phil. u. philos. Kiitik Bd. 152.)

Erkenntnis besessen habe. Dann muß dieser göttlichen Erleuchtung die Philosophie untergeordnet sein, was Avicenna in der Tat lehrt und zwar gerade an der Stelle, wo S. nach dem Lateinischen einen Rationalismus bei Avicenna sieht (S. 107f. Horten: Metaphys. Avic. 633). Als etwas „Überwundenes“ (S. 7, 8) erscheint ihm also der Islam nicht. Wenn man die jenseitige Vergeltung als eine geistige auffaßt, ist man ebensowenig ein Verteidiger der doppelten Wahrheit $(108,23)$, als wenn man den koranischen Ausdrücken ron ,, Augen Gottes" usw. einen übertragenen Sinn unterlegt. 
Bauer, Dr. Hans: Die Dogmatik al-Ghazálís nach dem zweiten Buche seines Hauptwerkes; Halle a. S. 1912; 77 S. $8^{0}$.

Zur Lösung der kulturhistorischen Aufgaben, die der Islam stellt, sind neben der philologischen Schulung die speziellsten Fachkenntnisse der einzelnen Kulturgebiete erforderlich. Die Geisterwelt des Gazali $1111 \uparrow$ vereinigt Theologie, Mystilk und Philosophie. Eine ausgezeichnete Vorschule für das Verständnis seiner Gedankengänge ist die christliche Scholastik, die der Verfasser der vorliegenden trefflichen Arbeit schulmäßig kennen gelernt hat. Daher ist er in der Lage, die schwierigen Originaltexte $z u$ verstehen und adäquat wiederzugeben. Er bietet in der genannten Arbeit weiteren Kreisen eine treffliche Übersetzung eines wichtigen Abschnittes aus dem Hauptwerke Gazalis: „Die Neubelebung der Religionswissenschaften“ dar, die er mit erläuternden Anmerkungen ausstattet. Die Stellung G.s zu den Philosophen und liberalen Theologen tritt dadurch deutlich hervor und damit zugleich auch seine Lebensaufgabe, eine umfassende Darstellung der herrschenden mystischen und religiösen Ideale des Islam zu geben. Da Gazali, der bekannte Gegneı Avicennas und Farabis, auch heute noch im islamischen Oriente maßgebend ist, besitżt diese Arbeit sogar ein aktuelles Interesse. Vgl. meine Bespr. ZDMG. 1913; 67, 563-65, wo gezeigt wird, das Gh. nicht so sehr Philosoph, sondern mehr Mystiker ist. Den Sinn wichtiger philosophischer Probleme übersieht er vollständig in der Formulierung seiner Gegner, der liberalen Theologen.

Taeschner, Franz: Die Psychologie Qazwínis. (Kieler Dissertation.) Tübingen 1912. $67 \mathrm{~S}$.

Kazwini 1283† ist der Plinius des islamischen Orients. In seiner Kosmographie gewährt er uns einen umfassenden Einblick in die Geisteskultur seiner Zeit. Durch die Arbeiten von Prof. G. Jacob, H. Ethé. Ruska und Ansbacher wurden wichtige Teile dieses Werkes, das den Titel trägt: „Die Wunder der Geschöpfe und die Denkmäler (,Spuren' $d$. h. Wirkungen der menschlichen Tätigkeit) der Länder" allgemein zugänglich gemacht. Taeschner verfolgt diese Aufgabe weiter, indem er in präziser und klarer Weise die psychologischen Abschnitte übersetzt und erläutert. Die Lehre von den Seelenkräften (vgl. auch H. Ethé: Morgenländische Studien, S. 125ff.: „Die Körper- und Geisteskräfte nach Kazwini") fußt durchaus auf Farabi und Avicenna, von denen manches wörtlich entlehnt zu sein scheint. Die Anordnung der achtzehn Tugenden, die ziemlich systemlos zu sein scheint, verrät keinen griechischen Zug. Die Art der Behandlung (Erläuterung durch Beispiele) ist die sufische, die in dem Tabakat immer wiederkehrt. Griechisch ist jedoch die Auffassung, daß die Tugend die goldene Mittelstraße zwischen zwei Lastern bedeute (32 Mitte), z. B. die Tapferkeit zwischen Feigheit und Tollkühnheit. In den Lehren über die eine Wunderkraft ausübenden Seelen treten platonische Gedanken deutlich zutage: In der himmlischen Welt (54f.) sind Archetypen vorhanden, die die Arten der irdischen darstellen. Sie sind also subsistierende Spezies, d. h. platonische Ideen, die die sublunarischen Dinge dirigieren, jeder Archetypus die ihm wesensgleiche Art. Durch Emanation aus diesen 
reinen Geistern der Himmel erkennen die reinen, lichtartigen Seelen auf der Erde die Wesenheiten der Dinge. Die Vorherbestimmung der Ereignisse durch die Sternenwelt geht so weit, daß in belanglosen Vorgängen z. B. dem Zerbrechen eines Glases $(65,6)$ oder dem Ausschütten von Wasser aus einem Schlauche $(67,11 \mathrm{ff}$.) Zukünftiges wie in Indizien enthalten ist - zweifellos ron den himmlischen Wesen mit Absicht hineingelegt. Der Wahrsager und Hellseher kann aus diesen Anzeichen die Ereignisse also voraussagen. Die naive Art und W.eise, wie die wundersüchtige Psyche des Menschen im Mittelalter auf die Betrachtung der Natur eingestellt war, findet dadurch eine lehrreiche Illustration. Zu einzelnen Punkten der Übersetzung möchte ich folgendes bemerken:

Unter Kainat 9,3 versteht der Araber die dem Werden und Vergehen (dem kaun und fasad vgl. ZDMG. 1911 Bd. 65 S. 539ff.) unterworfenen Dinge, also alles Vergängliche, Veränderliche, Gewordene im Gegensatz zu den Geistern, die in instanti durch Schöpfung entstehen d. h. nicht allmählich geworden sind und deshalb auch nicht in dem Sinne veränderlich sind wie die materiellen Dinge. Den Begriff „Existenzen“ würde er durch wugudat ausdrücken. - Alle aus Elementen gebildeten Dinge werden mauludat, hier muwalladat genannt, einschließlich der Metalle. Die wörtliche Übertragung dieses Terminus mit „die Erzeugten“ führt also leicht zu unadäquaten Vorstellungen, weshalb der im Mittelalter geprägte Terminus: "die Komposita“" $\mathrm{zu}$ bevorzugen ist. Hiss (ib. 8ff. "Gefühl") $=$ sinnliche (innere und äußere) Wahrnehmung. Kazwinis Stil charakterisiert sich durch viele Einschachtelungen und Schwerfälligkeit. T. hat dieses Kolorit beibehalten wollen. An vielen Stellen wünscht man jedoch im Interesse des Deutschen eine größere Zerlegung der Perioden. - "Zerfällt in Abschnitte“ $(12,2)=$ Text; elstreckt sich auf viele Dinge; ,Sinneswahrnehmungen“ $(12,1$ unt. $)=$ die bekannten Sinnesorgane; „er übertrug ihm die Macht über die geistigen Substanzen“" $(13,6)=$ Text: ,.er: üker trug den geistigen Substanzen die Macht über dasselbe“" d. h. das Gehirn, so daß der Verstand die Fähigkeiten desselben in seinen Intentionen beliebig verwenden kann; „Realitäten“ $(13,18)=$ eigentliche, innerste Wesenheiten; ,,der tierischen Richtung“ $(14,1)=$ freier: den tierischen Funktionen. Subjekt ist 15,6 wohl alma'lum in dem Sinne: Der Mensch als Objekt des Selbstbewußtseins, also das "Ich“ erkennt in allseitiger Weise alle erkembaren Dinge (der Außenwelt), ohne dabei ein Verlangen zu besitzen, sein eigenes Wesen (seine Innenwelt) zu begreifen. 'Amr (ib. 11 „Macht", Befehl) bezeichnet die Welt des Logos - wird wenigstens in diesem Sinne ron den Philosophen verstanden (nach dem syr. mìmar) - „Wir $(16,14 \mathrm{ff:})$ haben als Wahrheit gefunden (konstatiert), was unser Herr uns verheißen hat". Zu dem Gedichte Avicennas über die Seele (18f.) wurden die in ibn abì Usaibi'a (II 10) und tis'rasà'il (Konstantinopel 1298: Neun Abhandlungen Avicennas S. 129f.) vorliegenden Textzeugen wohl deshalb nicht herbeigezogen, weil sic nicht Wesentliches beitragen. Interessant ist, daß Avicenna Vers 17 auf eine Stelle des neuen Testamentes anspielt (I Kor. 2, 9), die von den Philosophen (z. B. Farabi: Ringsteine Nr. 22, meine Übersetzung S. 19, 22f.) häufig zitiert wird. Über die Geschichte dieses Zitates vgl. Macdonald: 
The life of Gazàli IAOS. 1899 Bd. 20 S. 76 A. 2. Die „tief eingewurzelte" (ràsiha) Disposition $(22,2)$ ist identisch mit dem habitus nach Aristoteles. „Man kam (22, 4f.; in A. 2 trifft Fleischer das Richtige) dazu (in der Definition der Charaktereigenschaft) die Bestimmung hinzuzufügen, daß sie tief in der Seele eingewurzelt sein muß, weil jemand z. B. nicht als freigebig (mit dem habitus der Freigebigkeit behaftet) bezeichnet wird, der nur aus bestimmten Anlässen ... die Handlung des Gebens ausübt“. „Man (ib. $9 \mathrm{ff}$.) stellte ferner die Bestimmung auf, daß die Handlungen mit Leichtigkeit erfolgen und zwar nur aus dem Grunde, weil ....". „Form" (ib. oft = hai'a) ist identisch mit: dispositio. Die Gegenüberstellung von Offenbarung und natürlichem Verstande (I 306, 1f., Ửbers. 22, 15ff.) erinnert an die theologischen Diskussionen über die Frage, ob es eine natürliche Sittlichkeit gebe, auch unabhängig von der Offenbarung. Kazwìni entscheidet sich hier für die liberale Thesis. - Der Mensch kann eine Tugend entweder absolut neu erwerben ,oder (23, $3 \mathrm{~min}$ nafsihi) an seiner Seele eine Anlage vorfinden, von der aus er zu einer anderen (Tugend, die dann àiso nur teilweise neu erworben ist) gelangt."

$\mathrm{Zu}$ bèachten ist, daß der Sufi Gunaid $910 \dagger$ I 307, 9 (Übers. 25, 4 unt.) schlechtweg als Pfau (Ta'ùs) bezeichnet wird (vgl. meine Besprechung von Frank: Scheich 'Adí in: Theolog. Literaturztg. $1912 \mathrm{Nr} .14 \mathrm{Sp} .418 \mathrm{f}$. und Nicholson: Kashf al-Mahjùb 189, 1). 'Atà' (wohl ibn 'Atà ib. 150, 9) ca. 930† und Muzàhid ca. 933 (vgl. Horten: philosophische Systeme 428, 8) waren die, Zeitgenossen dieses Tà'ûs ${ }^{1}$ ), der also nicht Sarràg (abû Nasr ca. 1050) gewesen sein kann, der ebenfalls diesen Beinamen hatte. - ,DeinVater war eị Renner, dessen Staub nicht gespalten wird (werden kann; 27, 1) - ein Bild, das auch von Yhilosophen gebraucht wird, die Unerreichbares geleistet haben sollen (vgl. Horten: Verzeichnis philos. Termini 370 Mitte). Der Gedanke ist wohl der, daß der erste Renner soweit voraus ist, daß der von ihm aufgewirbelte Staub sich senkt, bevor der folgende Renner dieselbe Stelle dẹ Bahn eIreicht hat. Von diesem wird alșo der aufgewirbelte Staub des ersten nicht „durchschnitten“. Daß del erste Renner, ,den Staub nicht durch seinen eigenen Körper teilt" (ib. A. 2) wird dem Gedanken des Unerreichbaren, der in diesem Bilde ausgedrückt sein soll, nicht genügend gerecht. 'Ad. 30 A. 4. Auch haffaftu 'àridaija bedeutet: ,,ich rasierte (wie hafaftu) meine beiden Wangen“" - nach dem stehenden Ausdrucke: hafif al'àridain = mit geschorenen beiden Wangen. - „Nicht habe ich vor" $(31,18)=$ Text: „Ich finde (,sehe") nicht, daß die ..." Ad. 37, 1. „Das Schlechte, was du nicht von mir kennst, überwiegt das, was du von mir kennst.“ Für „ungezählt“ $(39,12)$ lies: „ohne Rechenschaftsablegung“. - „Ich sah $(40,19)$ kein gutes Werk (an andern), das ich nicht bekannt machte, und kein schlechtes, das ich nicht (mit dem Mantel der Nächstenliebe) bedeckte". - Die Wahrhaftigkeit bosteht darin, daß die Zunge (die Rede) mit dem „Gewissen“ (der persönlichen Überzeugung,

1) Kazwìnì nennt (I 57, 9) den Gabriel den „Pfau“ der Engel. Ein geheimnisvoller Pfau (ib. I 165, 7 unt.) erscheint auf dem Berge Schakràn an Stelle eines Lichtes auf einem Leuchter. 
- nicht „dem Sinne" 41,10$)$ übereinstimmt. Ad. 316, 9: 'in wa scheint mir mit: „,wahrlich auch" (ihren Reiter; Übers. 50, 3 u. A. 1) wiederzugeben zu sein. - „Die Seelen der Wahrisager stehen in Kontakt mit den geistigen Substanzen $(58,10)$ “. Statt , unterbrochen werden“ lies $(59,18)$ : „endigen“:

Die mit großem Verständnisse ausgeführte Arbeit Taesçners zeigt, daß Kazwìn ohne ein Zurückgehen auf neue Handschriften nicht ganz übersetzbar ist und daß die Anmerkungen von Fleischer (handschriftl. Berlin Imp. c. notis mscr. 421) mit bewundernswerter. Treffsicherheit vielfach das Richtige finden. Die noch nicht übersetzten Teile Kazwìnis z. B. Botanik (I 245-301) und Anthropologie (I 322 - 368) usw. müßten nun auch bald eine ebenso vorzügliche Bearbeitung wie die vorliegende von Taeschner es ist, erfahren. Die Kenntnis-der islamischen Geisteskultur würde dadurch wesentlich gewinnen.

Luciani, J. D.: Les Prolégomènes Théologiques de Senoussi. Texte arabe et traduction française. Alger 1908. XII und $245 \mathrm{~S}$.

Sanùsì ist ein gutes Beispiel dafür, wie sehr griechisches Denken in die orthodoxeste Theologie des Islam eingedrungen ist. Als Basis seiner theologischen Auseinandersetzungen dient ihm die Lehre des Aristoteles von den Urteilen und Begriffen. Er ist sich bewußt, daß ohne diese logische Fundierung die Aufarbeitung der dogmatischen Gedankenwelt nicht reinlich vollzogen werden kann. Die innigste Verbindung von theologischem und philosophischem Denken ist ihm also Lebensbedingung der Theologie. Auch sonst ist sein Denken durchaus von der Philosophie beherrscht. Er sucht z. B. die Prinzipien, aus denen sich die Einteilung eines Gebietes a priori deduzieren läßt, z. B. die der vier Prädikate, die eine Handlung als ethische haben kann $(17,1)$. Die Definitionen sucht er in echt scholastisch-scharfsinniger Weise in allen ibren Teilen klarzustellen und gegen verwandte Gebiete abzugrenzen. Das fornelle Prädizieren ist ihm in Fleisch und Blut übergegangen. In den von ihm aufgestellten Thesen sind die Termini immer primo et per se zu verstehen. Dieses sind bereits die we`̀̀ntlichsten Eigenschaften des griechisch-philosophischen Denkens, die wir in der islamischen Theologie eingebürgert finden.

Die Tendenz dieser Prolegomena richtet sich gəgen die liberalen Theologen $(167,3)$ z. B. ihre Lehren, es gebe eine natürliche Sittlichkeit, die die menschliche Vernunft auch ohne die Offenbarung erkenne und aufstelle (21), - die sekundären, geschöpflichen (,empirischen“) Ursachen wirkten aus eigener Kraft unabhängig von Gott (111) - und die der Basrenser (169, 3), es gebe einen göttlichen Willen (den 'amr = Befehl und Wort, Logos), der außerhalb Gottes wie eine selbständige Substanz existiere und die Welt bilde (Demiourgos) - und besonders: Gott besitze keine Eigenschaften in der Form von realen Inhaerenzien (ma'ànì; 169, 5). Man könne von ihm nur die (rein logischen) Inhaerenzrerhältnisse (sifàt, mà'nawija 169, 6f.) aussagen, d. h. die Bestimmungen, die sich bei anderen Wesen aus jenen Eigenschaften ergeben in Gott aber mit der Wesenheit zusammenfallen. Die orthodoxe Thesis, Gottes Wesen sei das notwendige Sein, richtet sich ebenfalls gegen die mu'tazilitische: die 
innerste und speziellste Bestimmung Gottes sei die Ewigkeit $(173,6)$. Dabei ist jene orthodoxe Lehre der Begriffswelt der Philosophen entnommen. Mit Waffen, die den „Heiden“ (den Griechen) entlehnt sind, bekämpft Sanùsi also die eigenen Glaubensgenossen. - Bakillani wird als „der Imam der wahren Forscher" $(101,5)$ neben Gazàlì, Ràzì (Fahraddìn) und Guwainì ziticrt, was auf seine große Bedeutung schließen läßt. Auch über Nazzàm, Gahiz und Tumàma finden sich beachtenswerte Notizen. Die im folgenden zusammengestellten Bemerkungen betreffen einzelne Punkte der im ganzen vorzüglichen Übersetzung.

Mit ,adventicité" ( 3,3 unt.) kommt $L$. dem Begriffe des hudùt d. h. des zeitlichen Entstehens ('ihdàt Erschaffen in der Zeit) näher als seine Vorgänger mit: „nouveautéc. Das auf übernatürlicher Offenbarung beruhende Urteil auch der theologisch Ungebildeten nennt Sanùsì: hukm sar ì, während man unter ,jugement dogmatique (4 Mitte) ein solches der systematischen Theologie versteht; denn Dogmatik ist die intellektuelle Durcharbeitung der geoffenbarten Sätze, die S. mit jenem Ausdrucke nicht bezeichnen will. - Darùrì könnte in rein philosophischen Texten mit ,intuitif“ $(6,16)$ gut wiedergegeben werden. S. überträgt diesen Terminus aber auch auf das Gebiet der durch den bloßen Verstand nicht erreichbaren, sondern nur durch die koranische Offenbarung den Menschen zuteil gewordenen Lehren. Von einer Intuition des inneren und notwendigen Zusammenhanges von Subjekt und Prädikat kann bei diesen Sätzen, die eigentliche Mysterien sind, nicht gesprochen werden. Darùrì bedeutet dann also das mit unzweifelhafter Sicherheit als zum Bestande der Offenbarung gehörig Erkannte, das sich zu anderen Sätzen wie ein Prinzip verhält, also eine gewissere äußere Evidenz. Die innere Evidenz ist das darùrí'aklì. Ummahàt (10,2 übergangen) bedeutet wohl (in Zusammenstellung mit 'aba' Eltern) die sozialen Verbände oder die Autoritäten der Gemeinden. Mukallaf = rechtsfähiges Subjekt (dem deshalb. Pflichten auferlegt werden können) wird vielleicht etwas zu unbestimmt mit „,capable“" wiedergegeben. 'Ibàha d. h. Freistellung einer in sich indifferenten Handlung, sie zu tun oder zu unterlassen, sie für ethisch indifferent erklären, wird (14, 4 unt.) mit „Autorisation“ übersetzt. Es zeigt sich an diesen und.ähnlichen Fällen, daß die überaus knappe arabische Terminologie vielfach nicht mit derselben Kürze nachgeahmt werden kann. Man muß zu Umschreibungen greifen und das Prinzip der größten Kürze im Interesse der Deutlichkeit und adäquaten Wiedergabe aufgeben. Wie vortrefflich Luciani in das Verständnis des Textes und der Termini sonst eingedrungen ist, zeigt die Gleichsetzung von tard (gesetzmäßiges Verbundensein zweier Tatsachenreihen im Dasein) mit „correlation positive" und 'aks (Parallelismus und innere Verbindung der Dinge im Nichtsein) mit „correlation negative" (22 ob.). "Sans que cette relation (von Ursache und Wirkung; 32, 9) soit nécessaire" trifft den G'edanken von: ma'a sihhati - ttahalluf $(=$ „,dabei ist es möglich, daß die Wirkung hinter der Ursache zeitlich zurückbleibe“ oder: „daß ein Mißverhältnis zwischen beiden eintrete"). „Relation“ (34, 8 unt.) = Verknüpfung nach Art innerer Notwendigkeit, „créée“ (36, 10 unt.), ga'lij = auf willkürlicher Setzung $(\vartheta \dot{\varepsilon} \sigma \iota)$ beruhend „contraire" $(40,6$ unt.) = contradictoire (wie 
richtig 42, 9 = nakid), „règle imposée“ (42 Mitte) = willkürliche Annahme (vgl. IAP. 1897 S. 361), „,canonique“ (44, 12 unt.) = auf übernatürlicher Offenbarung beruhend (Gegensatz: auf die natürliche Vernunft und die Erfahrung sich stützend), „ni imposé, ni établi (44,9 unt.) = weder willkürlich aufstellbar, noch herstellbar, impénétrabilité" $(47,1$ unt.) = Räumlichkeit (der Körper ẉie 48,8), „antériorité éternelle“ $(48,1)=$ die" Notwendigkeit der ewigen Existenz, ,a posteriori“" $(48,11)=$ auf deduliktivem Wege, ,mystique“" $(60,5$ unt. $)=$ Realität der Tatsachen, l'optimisme logique“" $(112 \mathrm{Nr} .2)=$ die Lehre, daß die natürliche Vernunft (ohne Offenbarung) die sittliche Vortrefflichkeit einer Handlung erkennen und als Norm aufstellen könne (attahsìn al'aklij) - nach 117,1 unt. von den Brahmanen und (119, 5 unt.) liberalen Theologen vertreten, la faveur avec laquelle il considère" $(124,12)=$ weil er die unsichere Vermutung für richtig hält, auf die sich seine Spekulationen stützen, ,prèdominance et supériorité d'une chose sur une autre" $(150,3)=$ willkürliche Annahme (oder sophistische Argumentationsweise; tahakkum vgl. Horten: Verzeichnis philos. Termini S. 296, 7 unt.) und ursachloses Geschehen („Überwiegen der einen Wagschale über die andere ohne ausschlaggebendes Prinzip" $\left.{ }^{6}\right)$, „,indubitable et forcée“ $(160,3)=$ in denknotwendiger Evidenz, „attributs des idées" (162, 6 unt.) = diejenigen Eigenschaften Gottes, die reale Inhärenzien (ma'ànì vgl. ZDMG. Bd. 64 S. 391ff.) darstellen (z. B. das Wissen) im Gegensatze zu solchen, die Inhärenzverhältnisse bedeuten (sifàt ma'nawija z. B. das Wissendsein), „,indépendance“ $(164,10)=$ substanzielle Existenz (Gegensatz: inhärieren in einem Substrate), „correlation“ $(170,11)=$ innerlich notwendige Abhängigkeit und Unterordnung (nicht reziprokes Verhalten), „,monde moral“ $(172,10$ unt.) = die verborgene d. h. göttliche Welt (der Bestimmungen und Eigenschaften des Wesens Gottes), „modes d'action“ $(202,11)=$ die Beziehungen der Rede auf äußere Objekte (die bezeichnet werden sollen; ta'allukàt); „fausse dans sa conversion affirmative“ $(214,10)$ = falsch (ist eine Definition), wenn sie unkonvertiert verstanden und angewandt wird (weil sie nicht alle Dinge und Teile enthält, die das Definitum bilden), „fausse dans sa conversion negative“ $(216,4)=$ falsch (ist sie) als konvertierte, wenn sie mehr Dinge und Teile enthält, als das Definitum erfordert. Man kann sie dann nicht umkehren.) Die Definition muß alle Teile enthalten, wie L. 244 treffend entwickelt, also vollständig sein (dann wild sie als Gàmi' und muttarid, sahih attard bezeichnet) und auch nur diese, damit sie konviertierbar (mun'akis) ist und ,,ausschließend“ (màni) - „Intégrité $(226,6)=$ Treue $^{\text {" }}$ (in der Erfüllung der religiösen Pflichten, Gewissenhaftigkeit; Text 'amàna, nicht salàma). Demgegenüber bedeutet hijàna. Untreue, Gewissenlosigkeit. An die bekannten aristotelischen Gedanken über Wahrheit und Falschheit, die sich nur in der Aussage findet (Anfang der Logik) nicht in anderen sprachlichen Äußerungen, schließen sich die Lehren über habr (Prädikativ, Aussage) und 'inschà' (nichtprädikative Sprachäußerung, vielleicht gut mit „Exklamation“ wiederzugeben, Luciani: „inchoation" S. 209 und 241) an.

Die christliche Trinität faßt Sanùsì als Sein, Wissen und Leben Gottes $(7 \overline{7}, 7)$, eine Auffassung, die auch bei christlichen Theologen des islamischen 
Kulturbereiches 7. B. Paulus Bischof von Sidon (XIII. Jahrh. vgl. Philosophisches Jahrbuch 1906 S. 146, 5 und Horten: Systeme 103 A. 1) vertreten wurde. Die darauf folgende hochmütige Polemik gegen christliche Dogmen - eine Diskussion Ràzis $1210 \dagger$ mit einem Christen wird geschildert - zeigt deutlich, wie schr sich der Islam dem Christentum auch wissenschaftlich übcrlegen dünkte. Die Intoleranz Sanùsis tritt vielfach schroff hervor. Für die Geschichte der islamischen Theologie ließen sich noch manche Finzelheiten aus dieser reichen Quelle gewinnen. Das Mu'tazilitentum ist für die Zeit Sanùsis eine noch aktuelle Größe, die sich wie eine gefahrdrohende Macht erhebt. Daraus wird man jedoch nicht folgern dürfen, daß in jener Zeit noch rein mu'tazilitische Systeme in dem alten Sinne bestanden haben. Es handelt sich wolıl nur um Lehren dieser Richtung, die in späteren Systemen mit reichlicher Beimischung von griechischem Gedankengute fortleben. Luciani besitzt die genügende Stoffbeherrschung und Energie, um sich von den Fesseln der arabischen Ausdrucksweise und engherzig philologischer Betrachtung freizumachen und das Inhaltliche in den Vordergrund zu rücken. Auf diese Weise gelingt es ihm, eine klare und verständnisvelle Übersetzung zu liefern.

Wittmann, Dr. M.: Die Unterscheidung von Wesenheit und Dasein in der arabischen Philosophie. (Festgabe zum 60. Geburtstag von Cl.' Baeumker S. 34ff.)

Die islamische Philosophie bietet manche Aufschlüsse über scholastische Ideenbildungen, z. B. in der Lehre von den inneren Sinnen. Ein solcher Aufschluß ergibt sich auch für die Lehre über Wesenheit und Dasein. Während Plato Wesenheit und Individuum gegenüber. stellte, findet sich in der lateinischen Scholastik ziemlich unvermittelt das Begriffspaar: Wesenheit und Dasein. Der Übergang zwischen beiden vollzog sich in Farabi (Ringsteine 1). Er beginnt mit der platonischen Antithesis und ersetzt sodann die Individualität durch das Dasein, das für ihn zunächst das konkrete, dann aber auch das allgemeine Dasein ist. Damit ist die scholastische Lehre gegeben und zugleich der Boden für die großen Diskussionen im Islam über diese Frage (wie ein Reales, das Dasein, einem Unrealen, der Wesenheit, inhärieren könne). Sie führen mit der Zeit zu einer Identifizierung beider.

Eine andere Form des Gegensatzes zwischen beiden findet sich in den Systemen der islamischen Theologen. Die Wesenheit existiert als non-ens in schemenhafter Weise in einer vorwirklichen Welt (Lehre von der Realität des Nichtseienden rgl. das indische System der Vaisesika), aus der sie durch Hinzutritt des Daseins in die reale Wirklichkeit tritt. Vielach haftet sie der Wesenheit nur momentan an (vgl. das System der Sautrantika): Der Verfasser ist bemüht, diese offenbar indischen Lehren aus der griechischen Philosophie abzuleiten. Mir scheint, daß dieses Vergewaltigungen von Begriffen sind. Die nichtseienden aber doch ,realen“ Wesenheiten sollen die platonischen Ideen verschmolzen mit dem leeren Raume, dem "Nichtseienden" der Atomisten sein. So dankenswert also auch die klaren Ausführungen W.s betreffs des ersten Teiles sind, so wenig treffen sie das Richtige im zweiten.

(Schluß folgt im nächsten Heft.) 